\title{
Diagnóstico del trabajo en equipo en estudiantes de ingeniería en Chile
}

\author{
Rodrigo F. Herrera ${ }^{(1,2)}$, Felipe C. Muñoz ${ }^{(1)}$ y Luis A. Salazar ${ }^{(2)}$ \\ (1) Pontificia Universidad Católica de Valparaíso, Escuela de Ingeniería Civil, Avenida Brasil 2147, piso 3 , \\ Valparaíso - Chile. (e-mail: rodrigo.herrera@pucv.cl; fmunozlarivera@gmail.com) \\ (2) Pontificia Universidad Católica de Chile, Escuela de Ingeniería, Departamento de Ingeniería y Gestión \\ de la Construcción, Av. Vicuña Mackenna 4860, Macul, Santiago - Chile. (e-mail: lasalaza@uc.cl)
}

Recibido Mar. 29, 2017; Aceptado May. 29, 2017; Versión final Jun. 30, 2017, Publicado Oct. 2017

\begin{abstract}
Resumen
El objetivo de esta investigación es conocer cómo se prepara a los futuros ingenieros de Chile en el desarrollo del trabajo en equipo, y si existen diferencias entre distintas instituciones. El trabajo en equipo es una habilidad fundamental que todos los ingenieros deben tener en su carrera profesional. Es por este motivo que los centros de educación superior deben formar esta habilidad en los estudiantes de ingeniería. Dentro de los resultados principales de la investigación se encuentra que los estudiantes de ingeniería trabajan en equipo con compañeros de su misma carrera, pero no con compañeros de distinta carrera. Se encontró también que no existen diferencias entre los distintos centros de educación superior acerca de lo que estudiantes hacen cuando trabajan en equipo. Las prácticas que los estudiantes más hacen es comunicar y escuchar activamente a sus compañeros y la que menos hacen es colaborar efectivamente.
\end{abstract}

Palabras clave: trabajo en equipo; diagnóstico; estudiantes de ingeniería; universidades chilenas

\section{Diagnosis of the teamwork of engineering students in Chile}

\begin{abstract}
The objective of this research is to know how engineering students in Chile are prepared in the development of teamwork, and if there are differences between different institutions. Teamwork is a fundamental skill that all engineers must have in their professional career. It is for this reason that higher education institutions must train this skill in engineering students. Among the main results of the research it was found that engineering students work as a team with colleagues of the same career, but not with colleagues of different career. It was also found that there are no differences between the different higher education centers about what students do when working as a team. The practices that most students do is to actively communicate and listen to their peers and the least they do is effective collaboration.
\end{abstract}

Keywords: teamwork; diagnostic; engineering students; Chilean universities 


\section{INTRODUCCIÓN}

El trabajo en equipo es una competencia dinámica, multidimensional y con múltiples categorizaciones dada su complejidad, ésta supone la disposición personal y la colaboración con otros en la realización de actividades para lograr objetivos comunes, realizando actividades de intercambio de información, asignación de responsabilidades, resolución de conflictos, y una contribución a la mejora y desarrollo colectivo (Torreles et al., 2011). Dentro de los aspectos psicosociales que potencian la habilidad del trabajo en equipo se pueden destacar los siguientes: compromiso, toma de decisiones, gestión del tiempo, reconocimiento de roles, capacidad de integración, comunicación interpersonal, empatía, responsabilidad, reconocimiento de liderazgo y respeto al equipo (Barraycoa y Lasaga, 2010). El trabajo en equipo busca integrar las habilidades de cada uno de los miembros, generando canales múltiples de aprendizaje, basados en el compromiso y la confianza entre cada uno de estos integrantes (Raposo y Martínez, 2011).

En el proyecto Tuning se propone un listado de las habilidades genéricas que debe adquirir un estudiante a lo largo de su formación de pregrado, divididas en genéricas y específicas. El trabajo en equipo es una habilidad genérica de suma importancia, ya que con él se busca que los estudiantes interioricen actitudes básicas, tales como: transparencia, constancia, compromiso y respeto. Además, los estudiantes deben reforzar las habilidades de planificación y organización, así como otras relacionadas con la argumentación, consenso, toma de decisiones, resolución de conflictos, entre otras (Guitert et al., 2007). El trabajo en equipo de los estudiantes puede generar ciertas ventajas sobre el trabajo individual como medio parar superar algunas carencias formativas, por ejemplo, las dificultades para argumentar o para hablar en público. Las ventajas del trabajo en equipo van desde disminuir brechas relativas a la adquisición de conocimientos conceptuales o prácticos, hasta mejorar la actitud frente al aprendizaje (Cuadrado et al., 2012).

Dentro de las competencias profesionales demandadas por las empresas, en el caso de los ingenieros, se destacan la capacidad de comunicar, aprendizaje continuo, liderazgo, trabajar en equipo e innovación. Todas estas habilidades no están totalmente satisfechas, por lo que los futuros ingenieros se encuentran en una situación deficitaria en el mercado laboral (Marzo et al, 2006). Dentro de alguno de los resultados esperados de un estudiante de ingeniería que finaliza su proceso formativo de pregrado, se espera que cuente con la habilidad de trabajar en equipos multidisciplinarios (Allahverdi y Aldowaisan, 2015). Más aún, existen otros estudios que destacan que los empleadores de profesionales de ingeniería requieren tanto habilidades técnicas y blandas, tales como, la comunicación, trabajo en equipo, resolución de conflicto y liderazgo (Saad et al., 2013; Saad y Mayid, 2014; Crebert et al., 2007)

Trabajo en equipo para estudiantes de ingeniería es la capacidad para funcionar en equipos multidisciplinarios (Lingard, 2010; AACSB, 2013). Petrie (1976) sugiere que cada persona tiene un mapa cognitivo diferente y por tanto, dos estudiantes pueden ver cosas distintas ante un mismo acontecimiento. Hall (2005) plantea que ese mapa cognitivo se desarrolla como consecuencia de las experiencias educativas y socialización de los estudiantes de cada profesión. Por lo anterior, se deben proporcionar herramientas a los miembros del equipo para entender los mapas cognitivos del resto. Es clave que los establecimientos educacionales reconozcan y valoren las diferencias entre profesiones (Hinton Walker et al., 1998; Reese y Sontag, 2001) y establezcan vínculos de confianza y respeto que sustenten el trabajo colaborativo exitoso (Liedtka y Whitten, 1998). Trabajo en equipo facilita soluciones creativas a problemas difíciles (Drinka et al., 1996). En general a los diversos profesionales se les prepara para trabajar dentro de su propia profesión, no para comunicarse con individuos de otra profesión, lo que provoca barrera de vocabulario técnico, enfoques para resolver problemas y falta de entendimiento común de diversos temas y valores. (Hall, 2005). Las habilidades de cooperación, asertividad, responsabilidad, comunicación, autonomía y coordinación son esenciales para el eficaz trabajo en equipo (Norsen et al., 1995).

En el contexto de Chile se pueden distinguir tres tipos de instituciones que forman ingenieros, siendo estas: universidades tradicionales (UT), universidades no tradicionales (UNT) e institutos profesionales (IP). Se conoce como universidades tradicionales a las 25 universidades (de un total de 58) que pertenecen al Consejo de Rectores de Universidades Chilenas ( $\mathrm{CRUCH}$ ), éstas cuentan con aportes directos de financiamiento del estado por ser las de mayor tradición en el país. Por otra parte, las universidades no tradicionales que actualmente son 33, nacen después de la promulgación de la Ley General de Universidades en 1981 y no tienen dependencia estatal; por lo tanto, tampoco gozan de su financiamiento. Finalmente, los institutos profesionales son aquellas instituciones que pueden otorgar títulos profesionales y técnicos de nivel superior, pero no grados académicos, en la actualidad en Chile hay 43 institutos profesionales.

Si bien las instituciones anteriormente descritas declaran en sus perfiles de egreso que sus estudiantes tendrán la habilidad de trabajar en equipo, se desconoce en la práctica que tanto se les prepara en el 
desarrollo de esta habilidad, cómo se les prepara y si existen diferencias en la preparación entre instituciones. Lo anterior, implica que existe un desconocimiento de la condición de egreso de los estudiantes de ingeniería, sobre todo en lo que respecta a la habilidad del trabajo en equipo. Este trabajo busca responder, las siguientes preguntas de investigación: (1) ¿Qué ejecutan los estudiantes cuando se les solicita trabajar en equipo?, (2) ¿Cuál es la cantidad de veces que se les solicita a los estudiantes trabajar en equipo con estudiantes de la misma y otras carreras? y (3) ¿Existen diferencias entre lo que ejecutan los estudiantes de universidades tradicionales, no tradicionales e institutos profesionales?

\section{METODOLOGÍA}

Para responder a las preguntas de investigación planteadas, se utilizó como instrumento para medir las prácticas actuales de los estudiantes, cuando se les solicita trabajar en equipo, la encuesta propuesta por (Herrera et al., 2017), la cual se divide en tres secciones. En su primera sección, se clasifican a los estudiantes, según datos demográficos, de acuerdo al tipo de centro educacional; en su segunda sección, se mide la frecuencia a la que se les solicita trabajar en equipo a los estudiantes, especificando si son compañeros de la misma o diferente carrera; finalmente, en la tercera sección se analizan las prácticas a través de una escala de Likert de 1 a 5 (muy en desacuerdo $\{1\}$, en desacuerdo $\{2\}$, ni de acuerdo ni en desacuerdo $\{3\}$ de acuerdo $\{4\}$ y muy de acuerdo $\{5\}$ ) los criterios de coordinación, cumplimiento de tareas, resolución de conflictos, comunicación efectiva, liderazgo y colaboración. Cabe señalar, que este instrumento fue validado por un panel de expertos tanto del mundo académico como del profesional.

Si bien en esta oportunidad la encuesta fue aplicada a estudiantes de ingeniería de universidades chilenas, las preguntas expuestas en la Tabla 2 y Tabla 3 pueden ser realizadas a estudiantes de cualquier especialidad y de cualquier parte del mundo. En la Tabla 3 se incluyen prácticas de trabajo en equipo que deberían realizar los estudiantes cuando se les solicita este tipo de actividades.

Tabla 1: Instrumento de medición de tipo de centro de educación superior.

\begin{tabular}{|c|l|}
\hline \multicolumn{2}{|c|}{ 1. Indica en qué tipo de centro de educación superior estudias } \\
\hline a. & Universidad tradicional (UT) \\
\hline b. & Universidad NO tradicional (UNT) \\
\hline c. & Instituto Profesional (IP) \\
\hline
\end{tabular}

Tabla 2: Instrumento de medición de regularidad y tipo de compañeros en trabajo en equipo.

\begin{tabular}{|c|c|c|c|c|c|c|c|c|c|c|}
\hline \multicolumn{11}{|c|}{ 2. En tus últimos 10 cursos ¿en cuántos has trabajado en grupo o en equipo? } \\
\hline 0 & 1 & 2 & 3 & 4 & 5 & 6 & 7 & 8 & 9 & 10 \\
\hline \multicolumn{11}{|c|}{ 3. En tus últimos 10 cursos ¿en cuántos has trabajado con compañeros de la misma carrera? } \\
\hline 0 & 1 & 2 & 3 & 4 & 5 & 6 & 7 & 8 & 9 & 10 \\
\hline \multicolumn{11}{|c|}{ 4. En tus últimos 10 cursos ¿en cuántos has trabajado con compañeros de distinta carrera? } \\
\hline 0 & 1 & 2 & 3 & 4 & 5 & 6 & 7 & 8 & 9 & 10 \\
\hline
\end{tabular}

Tabla 3: Instrumento de medición de acuerdo respecto a afirmaciones afines al trabajo en equipo.

Con respecto a las siguientes afirmaciones contesta el grado de acuerdo que estás con cada una de ellas, en relación a tu práctica real y no la ideal. Siendo 1 Muy en desacuerdo y 5 Muy de acuerdo.

\begin{tabular}{|c|c|c|c|c|c|c|}
\hline Criterio & Afirmaciones & 1 & 2 & 3 & 4 & 5 \\
\hline Coordinación & $\begin{array}{l}\text { 5. Cuando desarrollo un trabajo en grupo/equipo, nos } \\
\text { dividimos las tareas equitativamente y definimos plazos de } \\
\text { cumplimiento }\end{array}$ & & & & & \\
\hline $\begin{array}{l}\text { Cumplimiento } \\
\text { de tareas e } \\
\text { hitos }\end{array}$ & $\begin{array}{l}\text { 6. Cuando desarrollo un trabajo en grupo/equipo, soy capaz } \\
\text { de cumplir con las tareas solicitadas en los plazos y calidad } \\
\text { previamente acordada }\end{array}$ & & & & & \\
\hline $\begin{array}{l}\text { Resolución de } \\
\text { conflictos }\end{array}$ & $\begin{array}{l}\text { 7. Cuando desarrollo un trabajo en grupo/equipo, soy capaz } \\
\text { de resolver conflictos que se generan al interior del equipo }\end{array}$ & & & & & \\
\hline
\end{tabular}


Tabla 3 (Continuación)

\begin{tabular}{|l|l|l|l|l|}
\hline Comunicación & $\begin{array}{l}\text { 8. Cuando desarrollo un trabajo en grupo/equipo, soy capaz } \\
\text { de comunicar adecuadamente mis ideas y escuchar } \\
\text { activamente a otros integrantes del equipo }\end{array}$ & & & \\
\hline Liderazgo & $\begin{array}{l}\text { 9. Cuando desarrollo un trabajo en grupo/equipo, soy capaz } \\
\text { de liderar al equipo para la consecución de los objetivos }\end{array}$ & & & \\
\hline Colaboración & $\begin{array}{l}\text { 10. Cuando desarrollo un trabajo en grupo/equipo, nos } \\
\text { reunimos a trabajar en conjunto para integrar, debatir y } \\
\text { analizar ideas }\end{array}$ & & & \\
\hline
\end{tabular}

La encuesta se aplicó a 220 estudiantes de ingeniería de educación superior en Chile, del total de estudiantes encuestados el 58,80\% pertenecen a universidades tradicionales, el $32,41 \%$ a universidades no tradicionales, mientras que el $8,80 \%$ pertenecen a institutos profesionales (Fig 1). Para resguardar la identidad de los encuestados, se utilizó como modelo de colección de datos la plataforma de formularios de Google. El posterior análisis de validación del instrumento se realizó por medio de un análisis de fiabilidad calculando el alfa de Cronbach (valor de 0,694), lo cual demostró una alta fiabilidad del instrumento realizado.

Se utilizan la prueba de Krustall Wallis se utiliza para determinar si las medianas de dos o más grupos difieren. Dado que es una prueba no paramétrica, se considera eficiente para estudiar muestras independientes, obtenidas de observaciones de distintos centros de educación superior.

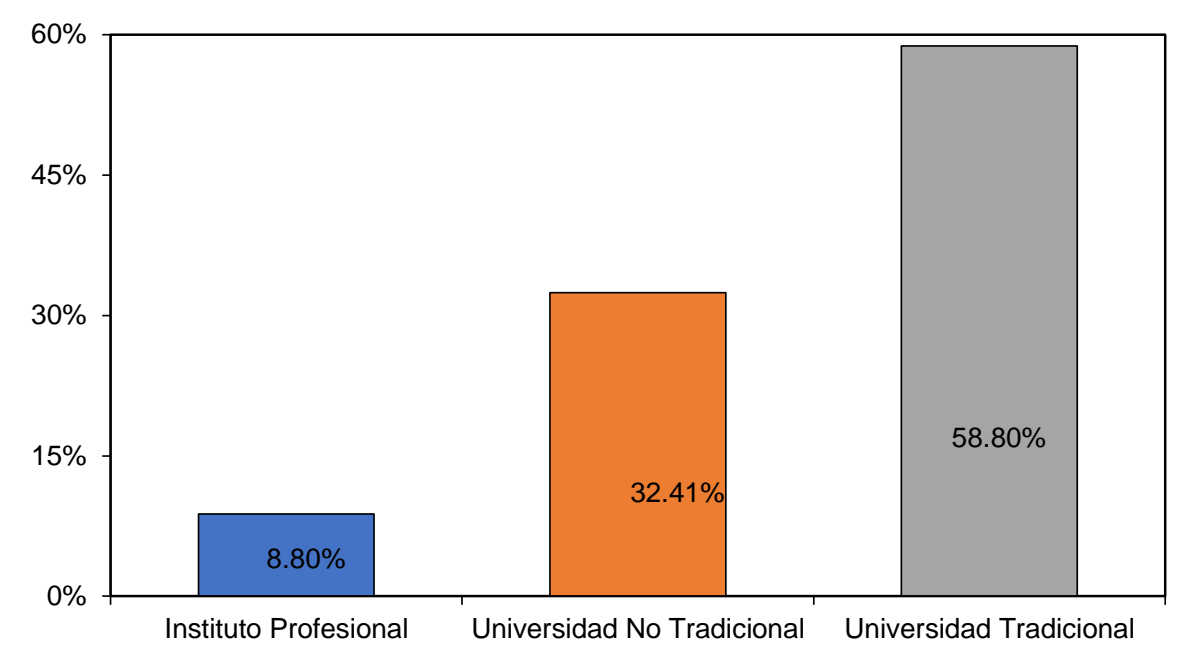

Fig. 1: Distribución de estudiantes encuestados.

\section{DISCUSIÓN DE RESULTADOS}

Para analizar los resultados se realizó un análisis descriptivo (medidas de tendencia central, variación y frecuencia) a nivel general y por tipo de centro educacional. Luego, para conocer si existen diferencias entre los estudiantes de distintos centros educacionales se utilizó el test de Krustall Wallis para comparar los tres tipos y el test $U$ de Mann Whitney para comparar entre pares de centros. La Fig. 2 muestra en el eje horizontal el porcentaje de cursos y en la vertical el porcentaje de estudiantes. Se individualizan 3 tipos de resultados; las barras azules denotan haber trabajado en equipo, las barras naranjas trabajo con estudiantes de la misma carrera y la barra gris con estudiantes de otras carreras.

Cuando se les consultó a los estudiantes respecto a si se les había hecho trabajar en grupo o en equipo en sus últimos 10 cursos rendidos (100\%), se observa que el $99,55 \%$ de ellos si ha trabajado de esta forma en por lo menos una asignatura. Al desglosar la cifra se tiene que un $80.63 \%$ de los alumnos ha trabajado en equipo en 5 o más de estos ramos, llegando al $22.52 \%$ de estudiantes que han trabajado de esta forma en la totalidad de sus últimas 10 asignaturas cursadas. Una tendencia similar se genera cuando a los estudiantes se les pregunta por el porcentaje de cursos que han trabajado con estudiantes de la misma carrera. Por otro lado, al explicitar si los compañeros de equipo han sido estudiantes de distinta carrera, un $40.54 \%$ de estudiantes declara no haber trabajado en equipo en ninguno de sus últimos 10 ramos y un $45.05 \%$ informan que lo han hecho en un máximo de 3 cursos. 


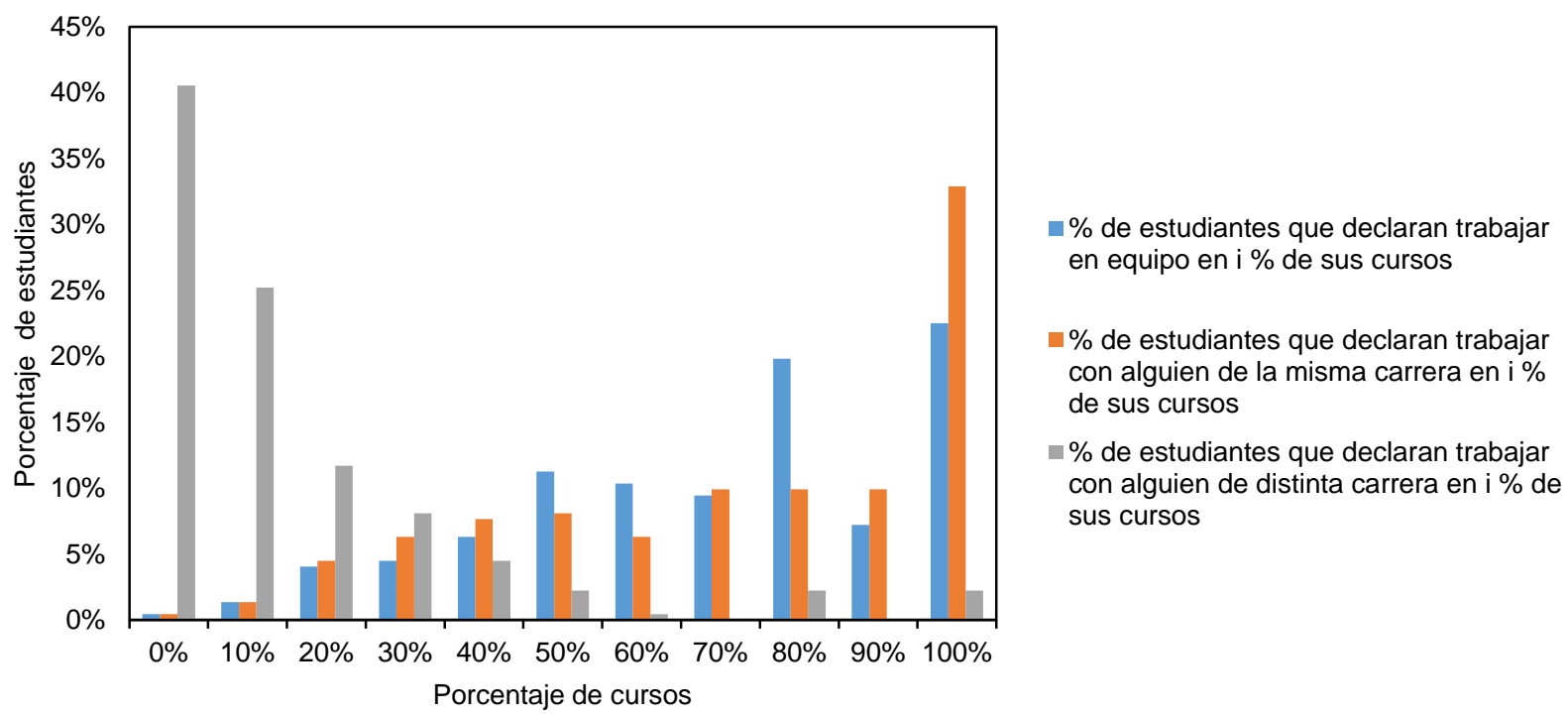

Fig. 2: Comparación de la cantidad de estudiantes que han trabajado en equipo en i cursos, desglosado en trabajo en equipo con estudiantes de la misma y distinta carrera.

Tabla 4: Medidas de tendencia central de los resultados

\begin{tabular}{|l|c|c|c|}
\cline { 2 - 4 } \multicolumn{1}{c|}{} & $\begin{array}{c}\text { \% de estudiantes que } \\
\text { declara trabajar en equipo } \\
\text { en i de sus cursos }\end{array}$ & $\begin{array}{c}\text { \% de estudiantes que declara } \\
\text { trabajar en equipo con alguien de la } \\
\text { misma carrera en i de sus cursos }\end{array}$ & $\begin{array}{c}\text { \% de estudiantes que declara } \\
\text { trabajar en equipo con alguien de } \\
\text { distinta carrera en i de sus cursos }\end{array}$ \\
\hline Media & 7.005 & 7.245 & 1.495 \\
\hline Mediana & 8.000 & 8.00 & 1.00 \\
\hline Moda & 10.00 & 10.00 & 0.00 \\
\hline Varianza & 6.274 & 7.553 & 4.558 \\
\hline SD & 2.505 & 2.748 & 2.135 \\
\hline
\end{tabular}

En base a las medidas de tendencia central se puede observar que en promedio los estudiantes realizan trabajo en equipo en 7.245 de sus últimos 10 cursos rendidos con estudiantes de la misma carrera, y en 1.495 de los cursos con estudiantes de otras carreras. Esto significa a que el desafío de trabajo en equipo se está enfrentando a nivel local en los programas de pregrado, pero no a nivel global, quedando al debe en el trabajo de equipos multidisciplinarios, tan solicitado por la industria. Luego, se solicitó detallar el grado de acuerdo respecto a algunas afirmaciones relacionadas con el accionar de los estudiantes frente al trabajo en equipo, en base a seis criterios. La Fig. 3 muestra la apreciación de los estudiantes ante cada uno de los criterios consultados para el total de estudiantes. Por otro lado, la Fig. 4 detalla los mismos resultados individualizados según el tipo de centro de educación superior.

Según los resultados, un $49.54 \%$ del total de estudiantes desarrolla colaboración efectiva en las instancias de trabajo en equipo; integrando, debatiendo y analizando ideas. Al desglosar esta percepción según el tipo de centro de educación superior, se observa que un $57.90 \%$ de los estudiantes de ingeniería de los IP trabaja colaborativamente en sus equipos de trabajo, seguido un $54.29 \%$ de los estudiantes de las UNT, dejando en último puesto a las UT con un $45.67 \%$ de los estudiantes. Respecto al criterio de liderazgo, un $73.15 \%$ del total de estudiantes consultados es capaz de liderar al equipo de trabajo y encaminarlo hacia la consecución de objetivos. En este sentido, un $77.95 \%$ de los estudiantes de ingeniería de UT se asume con líder de equipo, seguido por el $73.68 \%$ de los estudiantes de IP y por un $64.29 \%$ de los provenientes de UNT. Un $83.34 \%$ de los estudiantes consultados se declara que es capaz de comunicar adecuadamente sus ideas y escuchar activamente a otros integrantes del equipo. Quienes dan más relevancia a esta aptitud son los estudiantes de UT, llegando al $85.83 \%$ de ellos, seguidos por un $80.00 \%$ de los estudiantes de UNT y un $78.95 \%$ de IP.

Un $75 \%$ del total de estudiantes se declaran capaces de resolver los conflictos que se generan dentro del equipo de trabajo. El $78.95 \%$ de estudiantes de IP concuerdan con el desarrollo de dicha habilidad, seguidos por el $76.38 \%$ de estudiantes de UT y el $71.43 \%$ de estudiantes de UNT. Un 79,63 de estudiantes consultados es capaz de cumplir con los plazos y calidad del trabajo acordados. Al individualizar según tipo 
de casa de estudios, se aprecia un $84.25 \%$ de estudiantes de UT cumplen con dichos acuerdos, siguiéndole el $73.69 \%$ de estudiantes de IP y el $72.86 \%$ de estudiantes de UNT. Un $54.16 \%$ del total de estudiantes declara que, al desarrollar un trabajo en equipo, dividen las tareas entre los miembros y definen plazos de cumplimiento de las mismas. Lidera esta característica los estudiantes de IP, con un $63.16 \%$ de los consultados, seguido por el $55.12 \%$ de los estudiantes de UT y un $50.00 \%$ de estudiantes de UNT.

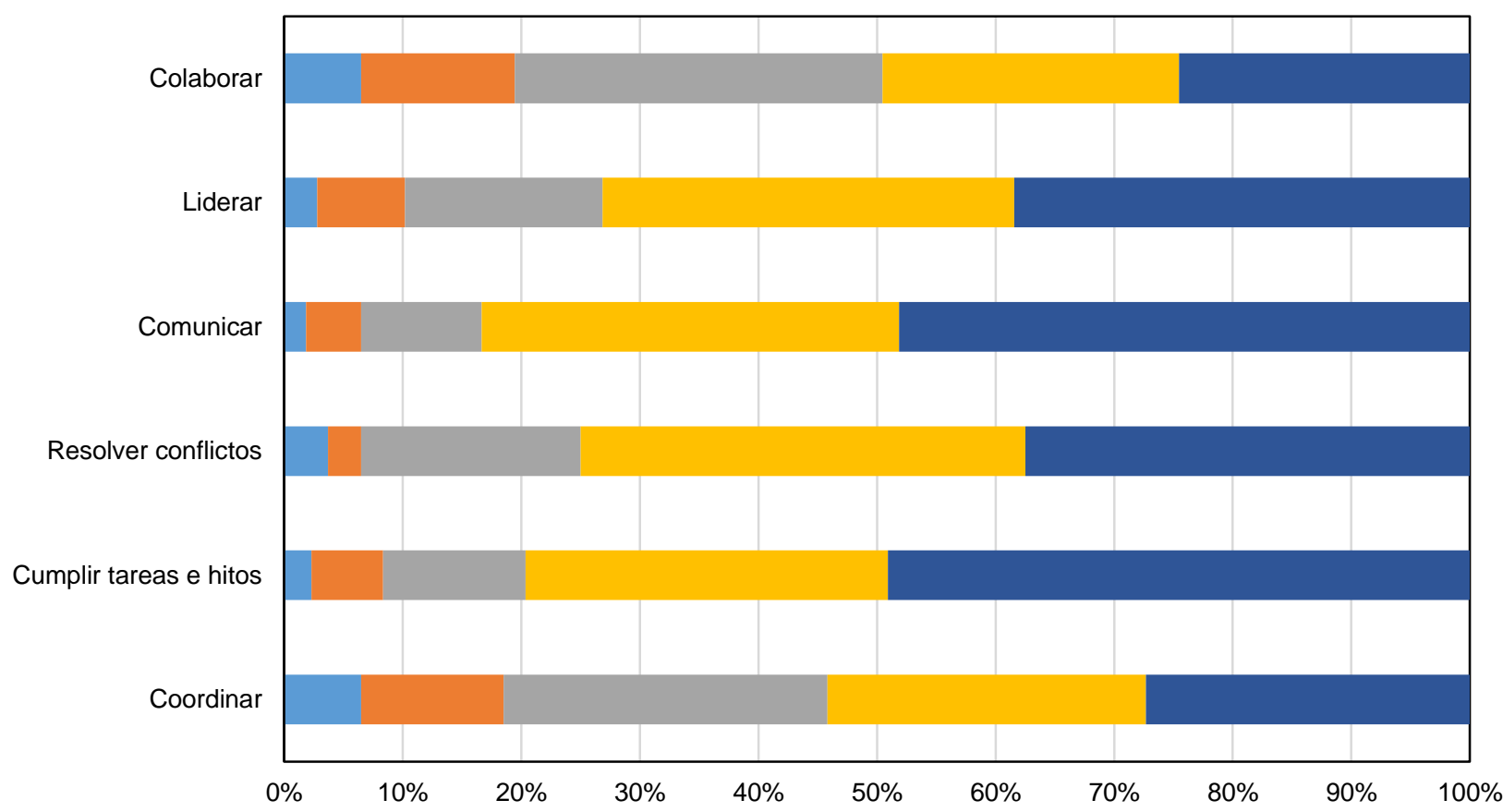

$\square$ (1) Muy en desacuerdo $\square$ (2) En desacuerdo $\square$ (3) Ni de acuerdo o desacuerdo $\square$ (4) De acuerdo $\square$ (5) Muy de acuerdo

Fig. 3: Importancia del trabajo en equipo según los estudiantes consultados.

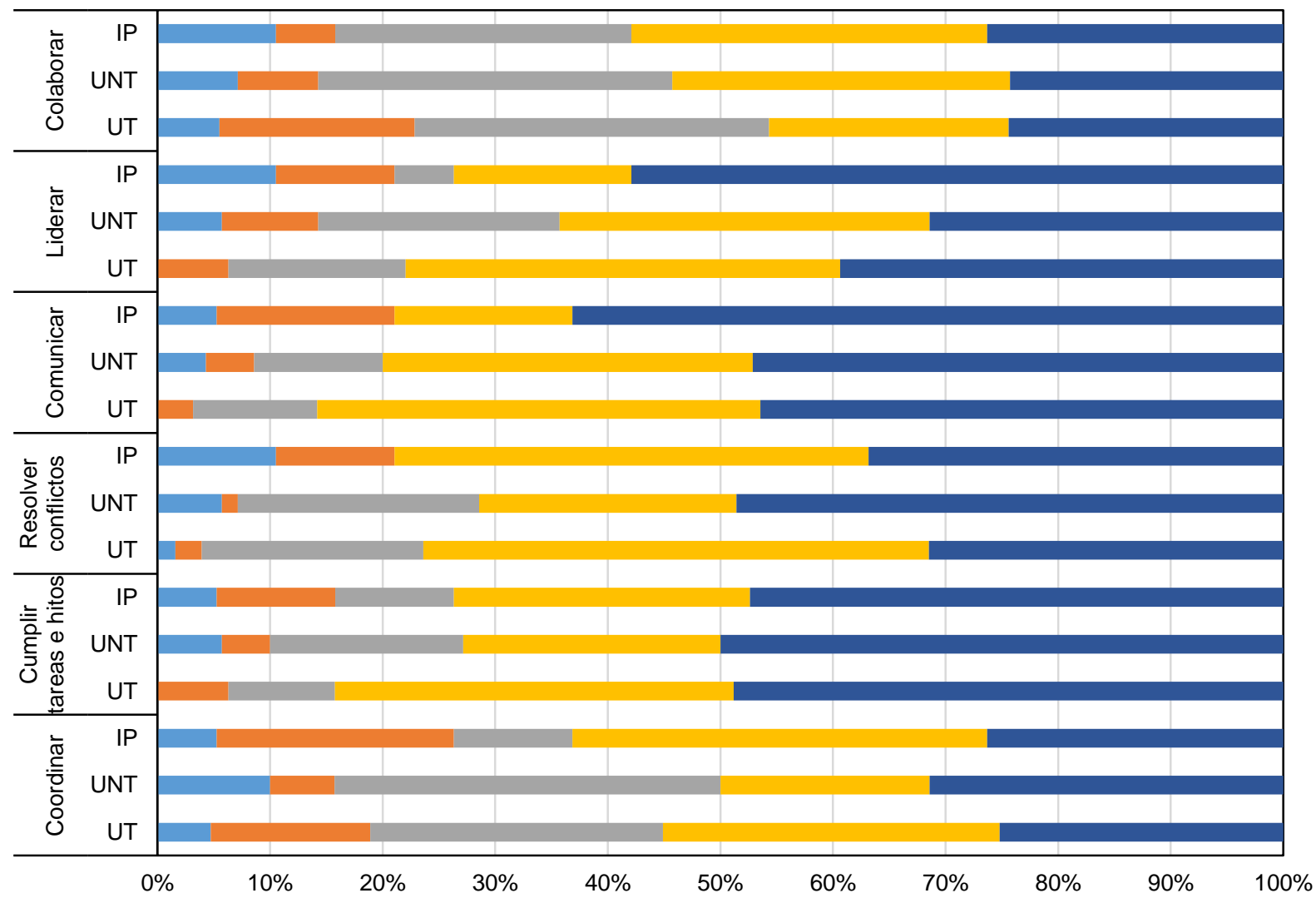

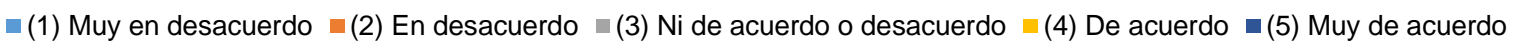

Fig. 4: Importancia del trabajo en equipo según los estudiantes consultados, desglosados según tipo de centro 
educacional de los estudiantes. 
Para identificar si existen diferencias entre lo que hacen los estudiantes de los distintos tipos de centros de educación superior al ser sometidos a trabajar en equipo, se utilizó la técnica $U$ de Mann-Whitney para comparar pares de centros y la prueba de Krustall Wallis para comparar los tres centros presentados. Con dicha técnica se verificó cada criterio con la que se presentó la habilidad de trabajo en equipo, realizando la siguiente formulación de hipótesis: $\mathrm{H}_{0}=$ No existe diferencia entre lo realizado por los estudiantes de los diferentes establecimientos de educación superior con respecto al criterio ${ }_{\mathrm{i}} \mathrm{H}_{1}=$ Existe diferencia entre lo realizado por los estudiantes de los diferentes establecimientos de educación superior con respecto al criterio

Se definió un nivel de significancia de un 0.05 y se obtuvo el p-valor para cara cada uno de los criterios establecidos, para saber si nos aceptamos las hipótesis $\mathrm{H}_{0} \circ \mathrm{H}_{1}$. Si el p-valor es mayor que el nivel de significancia no podemos rechazar la hipótesis nula y en caso contrario se puede rechazar la hipótesis nula y aceptamos $\mathrm{H}_{1}$. La Tabla 5 muestra los resultados de hipótesis y toma de decisión para el desarrollo de trabajo en equipo, ya sea con estudiantes de la misma o distinta carrera, comparando los diferentes centros de educación superior. La Tabla 6 muestra los resultados de hipótesis y toma de decisión para dichos planteles educacionales, pero respecto a cada una de las actividades desarrolladas durante el trabajo en equipo.

Tabla 5: p-valor para cada criterio y toma de decisión, respecto desarrollo de trabajo en equipo, ya sea con estudiantes de la misma o distinta carrera, comparando los diferentes centros de educación superior

\begin{tabular}{|c|c|c|c|}
\hline Criterio & $\begin{array}{l}\text { Tipo de centro de } \\
\text { educación superior } \\
\text { en comparación }\end{array}$ & $p$-valor & Decisión \\
\hline \multirow{4}{*}{$\begin{array}{l}\text { Trabajo en } \\
\text { equipo }\end{array}$} & UT-UNT-IP & 0.165 & $\begin{array}{l}\text { No existe diferencia entre lo realizado por los estudiantes de distintos } \\
\text { centros de educación superior }(\mathrm{H} 0)\end{array}$ \\
\hline & UT-UNT & 0.63 & $\begin{array}{l}\text { No existe diferencia entre lo realizado por los estudiantes de UT y de } \\
\text { UNT (H0) }\end{array}$ \\
\hline & UT-IP & 0.457 & $\begin{array}{l}\text { No existe diferencia entre lo realizado por los estudiantes de UT y de } \\
\text { IP }(\mathrm{H} 0)\end{array}$ \\
\hline & UNT-IP & 0.664 & $\begin{array}{l}\text { No existe diferencia entre lo realizado por los estudiantes de UNT y de } \\
\text { IP }(\mathrm{H} 0)\end{array}$ \\
\hline \multirow{4}{*}{$\begin{array}{l}\text { Trabajo en } \\
\text { equipo con } \\
\text { estudiantes } \\
\text { de la misma } \\
\text { carrera }\end{array}$} & UT-UNT-IP & 0.216 & $\begin{array}{l}\text { No existe diferencia entre lo realizado por los estudiantes de distintos } \\
\text { centros de educación superior }(\mathrm{H} 0)\end{array}$ \\
\hline & UT-UNT & 0.125 & $\begin{array}{l}\text { No existe diferencia entre lo realizado por los estudiantes de UT y de } \\
\text { UNT (H0) }\end{array}$ \\
\hline & UT-IP & 0.251 & $\begin{array}{l}\text { No existe diferencia entre lo realizado por los estudiantes de UT y de } \\
\text { IP }(\mathrm{H} 0)\end{array}$ \\
\hline & UNT-IP & 0.813 & $\begin{array}{l}\text { No existe diferencia entre lo realizado por los estudiantes de UNT y de } \\
\text { IP }(\mathrm{H} 0)\end{array}$ \\
\hline \multirow{4}{*}{$\begin{array}{l}\text { Trabajo en } \\
\text { equipo con } \\
\text { estudiantes } \\
\text { de distinta } \\
\text { carrera }\end{array}$} & UT-UNT-IP & 0.012 & $\begin{array}{l}\text { Existe diferencia entre lo realizado por los estudiantes de distintos } \\
\text { centros de educación superior }(\mathrm{H} 1)\end{array}$ \\
\hline & UT-UNT & 0.05 & $\begin{array}{l}\text { Existe diferencia entre lo realizado por los estudiantes de UT y de UNT } \\
(\mathrm{H} 1)\end{array}$ \\
\hline & UT-IP & 0.108 & $\begin{array}{l}\text { No existe diferencia entre lo realizado por los estudiantes de UT y de } \\
\text { IP }(\mathrm{H} 0)\end{array}$ \\
\hline & UNT-IP & 0.808 & $\begin{array}{l}\text { No existe diferencia entre lo realizado por los estudiantes de UNT y de } \\
\text { IP }(\mathrm{H} 0)\end{array}$ \\
\hline
\end{tabular}

Tabla 6: p-valor para cada criterio y toma de decisión, respecto desarrollo de trabajo en equipo para cada una de las habilidades y actividades desarrolladas durante el trabajo en equipo, comparando los diferentes centros

\begin{tabular}{|c|c|c|l|}
\hline Criterio & $\begin{array}{c}\text { Establecimientos } \\
\text { de educación } \\
\text { superior en } \\
\text { comparación }\end{array}$ & $p$-valor & \multicolumn{1}{|c|}{ Decisión } \\
\hline Colaboración & UT-UNT-IP & 0.616 & $\begin{array}{l}\text { No existe diferencia entre lo realizado por los estudiantes de distintos } \\
\text { centros de educación superior en lo que respecta a colaborar y } \\
\text { analizar el trabajo en conjunto (H0) }\end{array}$ \\
\cline { 2 - 4 } & UT-UNT & 0.398 & No existe diferencia entre lo realizado por los estudiantes de los tipos \\
\hline
\end{tabular}




\begin{tabular}{|c|c|c|l|}
\hline & UT-IP & 0.508 & $\begin{array}{l}\text { de centros de educación superior comparados en lo que respecta a } \\
\text { colaborar y analizar el trabajo en conjunto (H0) }\end{array}$ \\
\cline { 2 - 3 } & UNT-IP & 0.831 & \\
\hline
\end{tabular}

Tabla 6 (continuación)

\begin{tabular}{|c|c|c|c|}
\hline \multirow{4}{*}{ Liderazgo } & UT-UNT-IP & 0.111 & $\begin{array}{l}\text { No existe diferencia entre lo realizado por los estudiantes de distintos } \\
\text { establecimientos de educación superior en lo que respecta a liderar las } \\
\text { actividades del trabajo }(\mathrm{H} 0)\end{array}$ \\
\hline & UT-UNT & 0.054 & \multirow{3}{*}{$\begin{array}{l}\text { No existe diferencia entre lo realizado por los estudiantes de los tipos } \\
\text { de centros de educación superior comparados en lo que respecta a } \\
\text { liderar las actividades del trabajo }(\mathrm{H} 0)\end{array}$} \\
\hline & UT-IP & 0.539 & \\
\hline & UNT-IP & 0.177 & \\
\hline \multirow{4}{*}{ Comunicación } & UT-UNT-IP & 0.587 & $\begin{array}{l}\text { No existe diferencia entre lo realizado por los estudiantes de distintos } \\
\text { centros de educación superior en lo que respecta a tener una } \\
\text { comunicación efectiva entre los miembros del equipo }(\mathrm{H} 0)\end{array}$ \\
\hline & UT-UNT & 0.436 & \multirow{3}{*}{$\begin{array}{l}\text { No existe diferencia entre lo realizado por los estudiantes de los tipos } \\
\text { de centros de educación superior comparados en lo que respecta a } \\
\text { tener una comunicación efectiva entre los miembros del equipo }(\mathrm{H} 0)\end{array}$} \\
\hline & UT-IP & 0.568 & \\
\hline & UNT-IP & 0.401 & \\
\hline \multirow{4}{*}{ Conflictos } & UT-UNT-IP & 0.74 & $\begin{array}{l}\text { No existe diferencia entre lo realizado por los estudiantes de distintos } \\
\text { centros de educación superior en lo que respecta a resolver conflictos } \\
\text { durante la ejecución del trabajo }(\mathrm{H} 0)\end{array}$ \\
\hline & UT-UNT & 0.456 & \multirow{3}{*}{$\begin{array}{l}\text { No existe diferencia entre lo realizado por los estudiantes de los tipos } \\
\text { de centros de educación superior comparados en lo que respecta a } \\
\text { resolver conflictos durante la ejecución del trabajo }(\mathrm{H} 0)\end{array}$} \\
\hline & UT-IP & 0.96 & \\
\hline & UNT-IP & 0.628 & \\
\hline \multirow{4}{*}{ Tareas } & UT-UNT-IP & 0.784 & $\begin{array}{l}\text { No existe diferencia entre lo realizado por los estudiantes de distintos } \\
\text { centros de educación superior en lo que respecta a cumplir con las } \\
\text { tareas y los tiempos }(\mathrm{H} 0)\end{array}$ \\
\hline & UT-UNT & 0.605 & \multirow{3}{*}{$\begin{array}{l}\text { No existe diferencia entre lo realizado por los estudiantes de los tipos } \\
\text { de centros de educación superior comparados en lo que respecta a } \\
\text { cumplir con las tareas y los tiempos }(\mathrm{H} 0)\end{array}$} \\
\hline & UT-IP & 0.567 & \\
\hline & UNT-IP & 0.812 & \\
\hline \multirow{4}{*}{ Coordinación } & UT-UNT-IP & 0.962 & $\begin{array}{l}\text { No existe diferencia entre lo realizado por los estudiantes de distintos } \\
\text { centros de educación superior en lo que respecta a la coordinación del } \\
\text { trabajo }(\mathrm{H} 0)\end{array}$ \\
\hline & UT-UNT & 0.804 & \multirow{3}{*}{$\begin{array}{l}\text { No existe diferencia entre lo realizado por los estudiantes de los tipos } \\
\text { de centros de educación superior comparados en lo que respecta a la } \\
\text { coordinación del trabajo }(\mathrm{H} 0)\end{array}$} \\
\hline & UT-IP & 0.862 & \\
\hline & UNT-IP & 0.988 & \\
\hline
\end{tabular}

En variados casos de estudio se repiten fuertemente algunas habilidades cruciales en la formación universitaria en ingeniería ligadas al trabajo en equipo. Para el desarrollo del trabajo multidisciplinario real, estudiantes y profesionales del área hacen hincapié en la importancia del trabajo en equipo ligado a habilidades de responsabilidad, iniciativa y comunicación entre los pares (Fernández, et. al, 2010). En el área de ingeniería industrial, se destacan habilidades de liderazgo, comunicación y resolución de problemas como claves para el exitoso desarrollo de trabajo colaborativo (Jaca, et. al, 2016). El trabajar en equipo, liderando y comunicando efectivamente, son destrezas que deben ser evaluadas dentro de la formación en ingeniería, dada la relevancia que ellas cobran como habilidad profesional (Rojas, 2015).

\section{CONCLUSIONES}

En base a los resultados obtenido ha sido posible extraer las siguientes conclusiones: (i) Las prácticas que los estudiantes más desarrollan en instancias de trabajo en equipo son: capacidad de comunicar adecuadamente sus ideas y escuchar activamente a otros integrantes del equipo (83.34\% de los estudiantes), cumplir con los plazos y calidad del trabajo acordados $(79.63 \%$ de los estudiantes), resolver los conflictos que se generan dentro del equipo de trabajo $(75.00 \%$ de los estudiantes) y liderar al equipo de trabajo y encaminarlo hacia la consecución de objetivos (73.15\% de los estudiantes). En segundo plano queda la división de las tareas entre los miembros y definición de plazos de cumplimiento de las mismas $(54.16 \%$ de los estudiantes) y el desarrollo de una colaboración efectiva en las instancias de trabajo en equipo; integrando, debatiendo y analizando ideas (49.54\% de los estudiantes); (ii) Si bien existe una alta tasa de trabajo en equipo en los cursos de ingeniería (una media de 7 de los últimos 10 cursos rendidos por los estudiantes consultados), se prefiere la ejecución de dichas acciones con estudiantes de la misma carrera; un promedio de $72.45 \%$ de los cursos en desmedro de solo un $14.95 \%$ donde se han generado instancias de trabajo interdisciplinario con estudiantes de otras 
carreras; y (iii) Respecto a los distintos criterios consultados de acciones y habilidades relativas al trabajo en equipo, no existen diferencias significativas entre lo ejecutados por los estudiantes de universidades tradicionales (UT), universidades no tradicionales (UNT) y de institutos profesionales (IP).

Para lograr un exitoso desarrollo del trabajo en equipo, es fundamental determinar qué habilidades necesitan potenciar los estudiantes. Luego, se debe evaluar cómo implementar estas habilidades en las diferentes actividades académicas (trabajos, tareas y talleres) que se desarrollan en los diversos centros de educación superior del país. Para poner en práctica lo anterior, se hace gravitante desarrollar metodologías de enseñanza-aprendizaje orientadas al desarrollo de diversas habilidades que generen espacios de trabajo en equipos multidisciplinarios. Por lo anterior, los investigadores proponen realizar talleres de trabajo con estudiantes de diversas carreras afines a la Ingeniería como son Arquitectura, Derecho, Ingeniería Comercial, Construcción Civil, entre otras. Logrando de este modo, exhibir a los estudiantes a situaciones similares a las que se verán enfrentados en su quehacer profesional.

\section{AGRADECIMIENTOS}

Luis Arturo Salazar agradece el financiamiento de sus estudios de doctorados a Conicyt Chile (CONICYTPCHA/Doctorado Nacional/2016-21160819). Rodrigo F. Herrera agradece el financiamiento de sus estudios de doctorados a la beca VRI de la Pontificia Universidad Católica de Chile.

\section{REFERENCIAS}

AACSB International. Eligibility procedures and accreditation standards for business accreditation, www.aacsb.edu (2013)

Allahverdi, A. y Aldowaisan, T. Assessment of student outcomes of the Industrial and Management Systems Engineering programme at Kuwait Universit., Glob. J. Eng. Educ., 17, 3, 103-112 (2015)

Barraycoa J. y Lasaga, O. La Competencia De Trabajo En Equipo: Más Allá Del Corta y Pega, Vivat Acad., $111,66-70(2010)$

Crebert, G., Bates, M., Bell, B., Patrick, C.J. y Cagnolini, V. Developing generic skills at university, during work placement and in employment: Graduates' perceptions, High. Educ. Res. Dev., 32, 2, 147-165 (2007)

Cuadrado, C., Fernández, F.J., Fernández, M., Fernández-Pacheco, C., González, D. Lifante, I. y Moya, J. Técnicas de trabajo en equipo para estudiantes universitarios. En X Jornadas Redes de Investigación en Docencia Universitaria, Alicante (2012)

Drinka, T.J.K., Miller, T. F., y Goodman, B. M. Characterizing motivational styles of professionals who work on interdisciplinary healthcare teams, Journal of Interprofessional Care, 10, 51-61 (1996)

Fernández, L., Gutiérrez, J. e Hilera, J. Trabajo en equipo multinacional en ingeniería informática. VII Jornadas Internacionales De Innovación Universitaria, Madrid, 3-10 (2010)

Guitert, M., Romeu, T. y Pérez-Mateo, M. Competencias TIC y trabajo en equipo en entornos virtuales, RUSC Rev. Univ. y Soc. del Conoc., 4, 1, (2007)

Hall, P. Interprofessional teamwork: Professional cultures as barriers, Journal of Interprofessional Care, 19, $1,188-196$ (2005)

Herrera, R.F., Muñoz-La Rivera, F.C. y Salazar. L.A. The development's perception of teamwork competency in the formation of undergraduate engineering students, Glob. J. Eng. Educ., 19, 1, 30-35 (2017)

Hinton Walker, P., Baldwin, D, Fitzpatrick, J.J., Ryan, S., Bulger, R., DeBasio, y N., Hanson, C. Building community: Developing skills for interprofessional health, Nursing Outlook, 2, 88-89 (1998)

Jaca, C., Viles, E. y Zárraga-Rodriguez, M. Desarrollo de competencia de trabajo en equipo en un grado universitario. Memoria Investigaciones en Ingeniería, 14, 23-34 (2016)

Liedtka, J. M., y Whitten, E. Enhancing care delivery through cross-disciplinary collaboration: A case study, Journal of Health Care Management, 43, 185-203 (1998) 
Lingard, R.W. Teaching and assessing teamwork skills in engineering and computer science, Journal of Systemics, Cybernetics and Informatics, 18(1), 34-37 (2010) 
Marzo, M., Pedraja, M. y Rivera, P. Las competencias profesionales demandadas por las empresas: el caso de los ingenieros, Rev. Educ., 1, 643-661 (2006)

Norsen, L., Opladen, J., y Quinn, J. Practice model: Collaborative practice. Critical Care Nursing Clinics North America, 7, 43-52 (1995)

Petrie, H. G. Do you see what / see? Journal of Aesthetic Education, 10, 29-43 (1976)

Raposo, M. y Martínez E. La Rúbrica en la Enseñanza Universitaria: Un Recurso para la Tutoría de Grupos de Estudiantes, Form. Univ., 4, 4, 19-28 (2011)

Rojas, G. Medición de habilidades profesionales en estudiantes de ingeniería industrial de la universidad de Chile. Memoria para optar al título de Ingeniero Civil Industrial. Univ. de Chile, Facultad de Ciencias Físicas y Matemáticas, Depto. de Ingeniería Industrial. Disponible en: https://goo.gl/TR7Q79. Santiago de Chile (2015)

Reese, D. J., y Sontag, M. A. Successful interprofessional collaboration on the hospice team, Health and Social Work, 26, 169-175 (2001)

Saad, M.S., Robani, A., Jano, Z. y Makif, I.A. Employers' perception on engineering, information and communication technology (ICT) students' employability skills, Glob. J. Eng. Educ., 15 (1), $42-47$ (2013)

Saad, M.S. y Majid, I.A. Employers' perceptions of important employability skills required from Malaysian engineering and information and communication technology (ICT) graduates, Glob. J. Eng. Educ., 16, 3, 110-115 (2014)

Torrelles, C., Coiduras, J.L., Isus, S., Carrera, F. X., París, G. y Cela, J. M. Competencia De Trabajo En Equipo: Definición Y Categorización. Profr. Rev. Curric. y Form. del Profr., 15, 3, 329-344 (2011) 